\title{
WIRTSCHAFTLICHE AKTEURE UND \\ DIE DEUTSCH-FRANZÖSISCHE ZUSAMMENARBEIT: FORMEN, ZIELE, EINFLUSS
}

In seinem Jahresbericht 1963 nahm der Bundesverband der Deutschen Industrie (BDI) unter der Überschrift "Das weltpolitische Spannungsfeld" auch zum Elysée-Vertrag Stellung. Die deutsche Industrie, heißt es dort, "begrüßt die in diesem Vertrag dokumentierte Ausräumung des für die Geschichte beider Länder so verhängnisvollen Gegensatzes«. Der BDI habe von Anfang an die Politik der deutsch-französischen Aussöhnung nach Kräften gefördert und halte an dieser Auffassung genauso fest, "wie er unverdrossen bemüht bleibt, den Graben zuzuschütten ", der sich am 29. Januar 1963, also mit dem Abbruch der Beitrittsverhandlungen mit Großbritannien, in Brüssel aufgetan habe. Es sei wtief bedauerlich, da $B$ dieses Vertragswerk, das den uralten Gegensatz zwischen Deutschland und Frankreich endgültig auszuräumen« verspreche, durch die Gleichzeitigkeit der Ereignisse den "Eindruck eines inneren Zusammenhangs" mit dem Scheitern der Beitrittsverhandlungen erwecke. Darum müßten die europäischen Partner ebenso wie die Amerikaner davon überzeugt werden, da $\beta$ mit dem Vertrag "nur das bestätigt und befestigt wird, was bereits praktisch zur Pflege der Beziehungen zwischen den beiden Ländern geschieht und was das gute Verhältnis zu den übrigen Partnern nicht stört«.'

Dieses Zitat läßt eine eher gedämpfte Freude über den deutsch-französischen Vertrag vom Januar 1963 erkennen, verglichen etwa mit der Euphorie, die Bundeskanzler Adenauer zum Ausdruck brachte, als er den Vertrag in einer Fernsehansprache einen Tag nach der Unterzeichnung als »einzigartig in der Geschichte" würdigte. ${ }^{2}$ Wegen der gegen die Vereinigten Staaten von Amerika gerichteten Intentionen de Gaulles und - wenigstens zeitweise - auch Adenauers hegte der BDI sogar Bedenken, weil diese Intentionen die Einheit des Westens zu unterminieren drohten. Und mit Blick auf die deutsch-französische Aussöhnung hielt der

BDI-Jahresbericht 1962/63, S. 11.

Konrad ADENAUER, Erinnerungen 1959-1963. Fragmente, Stuttgart 1968, S. 212. 
Verband den Vertrag eigentlich für überflüssig, diene er doch hauptsächlich dazu, die bereits zuvor erreichte »Ausräumung des für die Geschichte beider Länder so verhängnisvollen Gegensatzes« zu dokumentieren. Das Gefühl, daß es dieses formellen Aktes gar nicht mehr bedurft hätte, herrschte auch auf französischer Seite vor - allerdings nicht etwa, weil die Unternehmer links und rechts des Rheins eine solche Aussöhnung ablehnten, sondern vielmehr, weil sie individuell oder unter Einschaltung von Organisationen unterschiedlichster Art seit 1947/48 auf verschiedenen Ebenen vergleichsweise eng kooperierten.

Dies traf auf die Gewerkschaften nicht zu. Ungeachtet ihrer internationalistischen Rhetorik waren sie wesentlich stärker auf ihr jeweiliges nationales politisches System und auf das Erreichen ihrer Ziele in der Auseinandersetzung mit ihren jeweiligen Regierungen und Industriellen ausgerichtet. ${ }^{3}$ Ein nicht zu unterschätzendes Hindernis für eine intensive Zusammenarbeit bildeten schließlich die Unterschiede in der politischen Orientierung. Im Gegensatz zu den sozialpartnerschaftlich-sozialdemokratisch geprägten Gewerkschaften in der Bundesrepublik gaben in der Gewerkschaftsbewegung jenseits des Rheins sozialistische und kommunistische Organisationen den Ton an. Kam es auch gelegentlich zu Treffen zwischen deutschen und französischen Gewerkschaftern - jene bemerkenswerte (außen-) politische Qualität, welche die Kooperation der Unternehmer auszeichnete, erreichten diese Kontakte zweifellos nicht. ${ }^{4}$ Aus diesen Gründen konzentrieren sich die folgenden Überlegungen auf die Unternehmer und ihre Organisationen.

Drei Aspekte der wirtschaftlichen und wirtschaftspolitischen Kooperation zwischen der Bundesrepublik und Frankreich sollen eingehender erörtert werden: Am Beginn steht ein Überblick über Akteure und Formen der Zusammenarbeit, danach werden gemeinsame und besondere, länderspezifische Ziele erörtert, ehe zum Abschluß der Versuch unternommen wird, den Einfluß der Unternehmerschaft auf die deutsch-französische Zusammenarbeit zu gewichten. Der zeitliche

Vgl. dazu grundsätzlich Bernhard EBBingHaus, Jelle VISSER, European Labor and Transnational Solidarity: Challenges, Pathways, and Barriers, in: Jytte KLAUSEN, Louise A. TiLlY (Hg.), European Integration in Social and Historical Perspective. 1850 to the Present, Lanham u.a. 1997, S. 195-221; am konkreten Beispiel Werner BÜHRER, Le Plan Schuman et les syndicats ouest-allemands, in: Andreas WILKENS (Hg.), Le Plan Schuman dans l'Histoire. Intérêts nationaux et projet européen, Brüssel 2004, S. 303-322.

4 Dieser Befund ergibt sich auch aus der umfassenden Dokumentation von Andreas Wilkens zu den deutsch-französischen Wirtschaftsbeziehungen, in der die Gewerkschaften nur am Rande vorkommen: Die Bundesrepublik Deutschland und Frankreich, Dokumente 1949-1963, hg. von Horst MÖLleR, Klaus HILDEBRAND, Bd. 2, Wirtschaft (=BDFD 2), bearb. von Andreas WILKENS, München 1997. 
Schwerpunkt liegt auf den knapp zwei Jahrzehnten zwischen 1945 und 1963, ergänzt durch einige Bemerkungen zu den Jahren davor und danach.

\section{Akteure und Formen der Zusammenarbeit}

Zwar hatten sich bereits zuvor einzelne Kontakte ergeben, ${ }^{5}$ doch läßt sich eine nachhaltige Steigerung erst seit Herbst 1947, also nach der Ankündigung des Marshall-Plans und dem Beginn der Verhandlungen über ein europäisches Wiederaufbauprogramm, konstatieren, als eine positive Vorentscheidung über die Zukunft der Industrie zumindest im westlichen Teil des besetzten Landes gefällt war. Die Protagonisten konnten dabei an eine Tradition deutsch-französischer Wirtschaftsbeziehungen anknüpfen, deren Anfänge sich bis ins 19. Jahrhundert zurückverfolgen lassen und die nach 1918 unter Bedingungen, die denen nach 1945 durchaus ähnelten, einen ersten Höhepunkt erlebten: Um das Reparationsproblem, insbesondere den Streit um deutsche Kohlenlieferungen nach Frankreich zu entschärfen, hatten sich nämlich nach dem Ersten Weltkrieg Schwerindustrielle beider Länder mit einigem Erfolg in direkten Verhandlungen um eine für beide Seiten akzeptable Lösung bemüht. Impulse zur Intensivierung der bilateralen Kontakte gingen auch von der 1926 gegründeten Internationalen Rohstahlgemeinschaft aus, die den Industriellen als institutionalisierter Rahmen für die Erprobung neuer Praktiken transnationaler wirtschaftlicher Interessenregulierung diente, sowie von dem auf Initiative des luxemburgischen Stahlindustriellen Emile Mayrisch entstandenen, gleichnamigen Komitee, das sich der ideellpolitischen Annäherung der "Erbfeinde « verschrieben hatte. ${ }^{6}$ Der daraus erwachsene Fundus an persönlichen Bekanntschaften und Erfahrungen dürfte dazu beigetragen haben, da $\beta$ selbst während der deutschen Besatzung kooperative Formen des geschäftlichen Umgangs nicht völlig verschwanden. Allerdings setzten sich

5 Vgl. Sylvie LEFÈVRE, Les relations économiques franco-allemandes de 1945 à 1955. De l'occupation à la coopération, Paris 1998, S. 215-217.

- Vgl. Wemer BÜHRER, Die Reorganisation der deutsch-französischen Wirtschaftsbeziehungen nach 1918 und nach 1945, in: Ilja MIECK, Pjerre GUILLEN ( $\mathrm{Hg}$.), Nachkriegsgesellschaften in Deutschland und Frankreich im 20. Jahrhundert, München 1998, S. 67-81, hier S. 68-76; zum »Mayrisch-Komitee» vgl. Fernand L'HuILliER, Dialogues Franco-Allemands 19251933, Paris 1971. 
die französischen Industriellen dabei dem Vorwurf der Kollaboration aus ${ }^{7}$ - ein Grund dafür, daß sie in den ersten Jahren nach Kriegsende sorgfältig darauf achteten, die Wiederbelebung der Beziehungen nicht zu forcieren.

$\mathrm{Zu}$ denen, die im Herbst 1947 den Gesprächsfaden wiederaufzunehmen versuchten, gehörten Vertreter der de Wendel-Gruppe, des luxemburgischen ARBED-Konzerns und der Kölner Bankier Robert Pferdmenges, ein enger Vertrauter Konrad Adenauers; letzterer konnte sich bei seinen Sondierungen der Unterstützung einflußreicher Ruhrindustrieller wie Hermann Reusch von der Gutehoffnungshütte und Günter Henle von Klöckner gewiß sein. Schon im März 1948 vermeldete Reusch zufrieden, daß es keine Schwierigkeiten bereite, mit den »von früher her befreundeten französischen Wirtschaftskreisen erneut Fühlung aufzunehmen ${ }^{8}{ }^{8}$ Aus solchen unregelmäßigen Treffen wurden im Herbst 1948 regelmäßige, institutionalisierte Kontakte: Auf französischer Seite übernahm zunächst die "Association pour le Commerce et l'Industrie Française en Allemagne" (ACIA) die Führungsrolle, im Frühjahr 1949 rückte die "Association Française pour les Relations Economiques avec l'Allemagne" (AFREA) an ihre Stelle. Auf deutscher Seite entstand im November 1948 in Frankfurt auf Initiative der Industrie- und Handelskammern des Rhein-Main-Gebietes der "Arbeitskreis Frankreich «, wenig später konstituierte sich in Düsseldorf ein "Studienausschuß für die deutsch-französischen Wirtschaftsbeziehungen ", ehe Mitglieder dieser beiden Zusammenschlüsse im September 1949 die "Deutsche Vereinigung zur Förderung der Wirtschaftsbeziehungen mit Frankreich « (DEFRA) gründeten. Zu diesem Zeitpunkt hatte bereits ein erstes »offizielles« Treffen zwischen dem Vorstand der AFREA und Mitgliedern des Frankfurter und des Düsseldorfer Kreises stattgefunden. Die Zusammenarbeit dieser beiden Organisationen mündete schließlich im Juni 1955 in die Gründung der deutsch-französischen Handelskammer. ${ }^{9}$

An diesen Zusammenkünften nahmen zwar auch Vertreter des 1946 aus der Taufe gehobenen "Conseil National du Patronat Français" (CNPF) und des im Entstehen begriffenen deutschen Pendants, des BDI, teil, doch bemühten sich die beiden Spitzenverbände schon bald um eine exklusivere organisatorische Platt-

7 Vgl. Annie LACROIX-RJZ, Industriels et banquiers français sous l'occupation. La collaboration économique avec le Reich et Vichy, Paris 1999; Monika RIESS, Die deutsch-französische industrielle Kollaboration während des Zweiten Weltkrieges am Beispiel der RENAULTWerke (1940-1944), Frankfurt a. M. 2002.

8 Aufzeichnung Kroll, 8.3.1948, Hauptstaatsarchiv Düsseldorf, NW 53, 113, Bl. 103-105.

9 Vgl. auch Werner BüHRER, Wegbereiter der Verständigung. Deutsch-französische Industriellenkontakte 1947-1955, in: Revue d'Allemagne, 23 (1991) 1, S. 73-86. 
form für den als notwendig erachteten, kontinuierlichen Meinungsaustausch. Diese Bemühungen wurden dadurch begünstigt, daß führende Repräsentanten des BDI und des CNPF als Mitglieder europäischer bzw. internationaler Industriellenverbände häufig zusammenkamen - beispielsweise im "Rat der Europäischen Industrieverbände" (oder "Conseil des Fédérations Industrielles de l'Europe«, CIFE), der die Interessen der Industrie gegenüber der »Organisation for European Economic Co-operation « (OEEC) vertrat, oder in der "Union der Industrien der sechs Schumanplanländer«, einem Vorläufer der noch heute bestehenden »Union des Confédérations de l'Industrie et des Employers d'Europe (UNICE). Im November 1951 etablierten die Spitzen der Industrie bzw. der Dachverbände beider Länder schließlich ein deutsch-französisches »Industrie-Komitee«, nach einem der französischen Initiatoren auch »Métral-Komitee« genannt, das künftig mindestens einmal pro Jahr tagte. Das erste Treffen in Düsseldorf zeichnete sich durch einen hochrangigen Teilnehmerkreis aus - darunter Fritz Berg und zwei weitere Präsidiumsmitglieder des BDI auf deutscher, CNPF-Präsident Georges Villiers und sein Stellvertreter Pierre Ricard auf französischer Seite - und signalisierte schon allein dadurch den Führungsanspruch der beiden Spitzenverbände.

Die im großen und ganzen reibungslose und enge Kooperation zwischen BDI und CNPF war zweifellos auch eine Folge der guten persönlichen Beziehungen zwischen den beiden Präsidenten. Angesichts ihrer gegensätzlichen Karrieren mag diese wechselseitige Sympathie auf den ersten Blick überraschen: Berg, seit 1941 Alleininhaber eines mittelständischen Betriebs, der sich auf die Drahtherstellung und -verarbeitung spezialisiert hatte, NSDAP-Mitglied von 1937 bis zum Kriegsende, war unter anderem als Leiter der Fachgruppe Fahrrad- und Kraftradteile-Industrie sowie, seit Anfang 1942, auch der Fachgruppe Metallmöbel und Matratzen ein "gut funktionierendes Rädchen im Getriebe des nationalsozialistischen Wirtschaftsystems « gewesen; ${ }^{10}$ Villiers hingegen, gleichfalls in der mittelständischen metallverarbeitenden Industrie tätig, 1941 bis 1943 von der Vichy-Regierung ernannter Bürgermeister von Lyon, war wegen Verbindungen zur Résistance vom Sommer 1944 bis zur Befreiung im KZ Dachau interniert gewesen." Dennoch, die »Chemie« zwischen den beiden stimmte: Ihre Zugehörigkeit zur mittelständischen Industrie dürfte dabei ebenso eine Rolle gespielt haben wie ihre intellektuelle Mittelmäßigkeit, die manchen der „Großen« aus traditionsreichen Unternehmerdynastien gelegentlich die Nase über solche "Empor-

10 Achim SCHULTE-GoeBel, Fritz Berg 1901-1979: Unternehmer und Industriepräsident in der Adenauer-Ära, unveröffentlichte Diss. Düsseldorf 1999, S. 10-20, hier S. 20.

1 Vgl. Henry W. EhrmanN, Organized Business in France, Princeton 1957, S. 132-135; Henri WEBER, Le parti des patrons. Le CNPF (1946-1986), Paris 1986, S. 76. 
kömmlinge« rümpfen ließ und bei den beiden Inferioritätsgefưhle auslöste, sowie ihr gemeinsamer Glaube an das "freie Unternehmertum " und ihre »europäische" Passion und Mission. ${ }^{12}$ Jedenfalls spielte sich schon Anfang der 1950er Jahre eine direkte und gute, institutionalisierte Zusammenarbeit zwischen BDI und CNPF ein, die ihren Ausdruck beispielsweise auch in der Rede Villiers' auf dem »Europatag« des BDI im Oktober 1952 oder auf dessen Mitgliederversammlung im Mai 1955 fand. Insofern verwundert es nicht, daß die Spitzen des BDI und des CNPF die Aufforderung des französischen Ministerpräsidenten Pierre Mendès France anläßlich seines Treffens mit Bundeskanzler Adenauer in La Celle-St. Cloud im Oktober $1954,{ }^{13}$ die Verbände sollten ihre Beziehungen wlebendiger" gestalten, nicht auf sich bezogen, aber dafür sorgen wollten, daß die »bereits bestehende Zusammenarbeit zwischen den industries homologues « intensiviert werde; ferner sollten die bilateralen Gespräche durch vorherige Konsultation interessierter Firmen und Verbände noch besser vorbereitet werden. ${ }^{14}$ Im übrigen war der BDI sogar bereit, auf die Mitte der 1950er Jahre anscheinend wwachsende Besorgnis der französischen Industrie über das $A$ usma $B$ und Tempo der Wiederherstellung der deutschen Produktionskapazitäten « Rücksicht zu nehmen und die nunbestreitbar hervorragenden Leistungen der deutschen Industrie nach außen nicht zu betont herauszustellen . $^{15}$

Obwohl die Intensität ihrer Kontakte nicht das Niveau der Verbindung zwischen BDI und CNPF erreichte, arbeiteten zu diesem Zeitpunkt auch manche Branchenverbände schon seit einigen Jahren zusammen. Vor allem die Stahlverbände der Bundesrepublik und Frankreichs bemühten sich, nicht zuletzt unter dem Druck der Verhandlungen über den Schuman-Plan, um eine Klärung und Abstimmung ihrer Positionen und Zielsetzungen. Die Treffen beispielsweise zwischen den Organisationen der Chemie-, der Automobil- und der Elektroindustrie wurden gleichfalls allmählich zahlreicher. Der Einsatz der Industrie- und Han-

$12 \mathrm{Vgl}$. zum letzten Punkt Marine MOGUEN-TOURSEL, L'ouverture des frontières européennes dans les années 50. Fruit d'une concertation avec les industriels?, Brüssel 2002, S. 35.

13

Vgl. Andreas WILKENS, Das Programm von La Celle-St. Cloud. Der Ausbau der deutschfranzösischen Wirtschaftsbeziehungen 1954-1957, in: Revue d'Allemagne et des Pays de langue allemande, 25 (1993) 4, S. 565-580 (Themenheft zu den deutsch-französischen Wirtschaftsbeziehungen im 20. Jahrhundert unter der Leitung von Raymond Poidevin).

14 Protokoll Präsidialsitzung vom 26.1.1955, BDI-Archiv, HGF Pro, 4; Berg an Adenauer vom 11.5.1955 (mit Anlage), in: BDFD 2 (Anm. 4), S. 1022-1026. Der deutsch-französische Wirtschaftsausschuß, der als Folge des Treffens zwischen Mendès France und Adenauer gegründet wurde, sollte nach Ansicht der beiden Verbände ein »reiner Regienungsausschuß « bleiben, so der BDI-Jahresbericht 1956/57, S. 30. Nennenswerte Bedeutung erlangte dieses Gremium jedoch nicht.

15 Protokoll Präsidialsitzung vom 13.9.1954, BDI-Archiv, HGF Pro, 3. 
delskammern zugunsten einer Institutionalisierung ihrer Verbindungen und die beharrliche Arbeit, welche AFREA und DEFRA leisteten, trug mit der Gründung einer deutschen Handelskammer in Frankreich, einer französischen in der Bundesrepublik und schließlich, wie erwähnt, einer gemischten deutsch-französischen Handelskammer im Sommer 1955 in Paris ebenfalls Früchte. Dagegen kam die Zusammenarbeit zwischen den Unternehmen nur langsam in Gang: Der erste spektakuläre Fall nach Kriegsende ereignete sich 1953, als ein französisches Konsortium vom Flick-Konzern die Aktienmehrheit der Harpener Bergwerks AG erwarb. Ein qualitativer Sprung fand indes erst nach der Konstituierung der Europäischen Wirtschaftsgemeinschaft (EWG) statt, als mit der schrittweisen Verwirklichung des Binnenmarktes für die Unternehmen neue Möglichkeiten und Zwänge zur Kooperation entstanden. ${ }^{16}$ Erwähnt zu werden verdient zuletzt noch die 1949 zunächst auf multilateraler Basis aufgenommene, wenngleich nicht gänzlich reibungslos funktionierende Verbindung zwischen dem Deutschen Bauernverband und den französischen Agrarverbänden. ${ }^{17}$

Spätestens Mitte der 1950er Jahre existierte somit ein dichtes Netz institutionalisierter bilateraler und multilateraler Kontakte zwischen der deutschen und französischen Wirtschaft, das durch informell-individuelle Zusammenkünfte einzelner Industrieller noch engmaschiger wurde. Zusätzliche Organisationen, wie sie manche Politiker gelegentlich wünschten, waren also überflüssig und hätten möglicherweise eher einen gegenteiligen, die Zusammenarbeit erschwerenden Effekt gehabt.

$16 \mathrm{Vgl}$. mit zahlreichen Beispielen für die Zusammenarbeit auf unterschiedlichen Ebenen BDFD 2, S. 950-1046; ferner Gottfried PLUMPE, Ansätze zur Zusammenarbeit zwischen der deutschen und französischen Chemieindustrie vor und nach dem Weltkrieg, in: Yves COHEN, Klaus MANFraSS (Hg.), Frankreich und Deutschland. Forschung, Technologie und industrielle Entwicklung im 19. und 20. Jahrhundert, München 1990, S. 224-233; Thomas HER2IG, Elektroindustrie und Energieverbund zwischen Deutschland und Frankreich von der Jahrhundertwende bis in die 50er Jahre, in: ibid. S. 289-301; Patrick FRIDENSON, Les relations entre les industries automobiles française et allemande des années 1880 aux années 1960, in: ibid. S. 334-342; Sylvie LEFÈVRE, Les sidérurgistes français propriétaires de charbonnages dans la Ruhr (1945-1954), in: Andreas WILKENS (Hg.), Die deutsch-französischen Wirtschaftsbeziehungen 1945-1960, Sigmaringen 1997, S. 237-247, hier S. 244-246; Jean-François ECK, Les entreprises françaises face à l'Allemagne de 1945 à la fin des années 1960, Paris 2003.

$17 \mathrm{Vgl}$. Gilbert NOEL, France, Allemagne et "Europe Vert«, Bem 1995; Guido THIEMEYER, Vom »Pool Vert« zur Europäischen Wirtschaftsgemeinschaft. Europäische Integration, Kalter Krieg und die Anfänge der Gemeinsamen Europäischen Agrarpolitik, München 1999. 


\section{Separate und gemeinsame Ziele}

Zwischen der deutschen und französischen Wirtschaft hatte sich seit dem später 19. Jahrhundert ein enges, wechselseitig vorteilhaftes Austauschverhältnis entwickelt, das allerdings infolge politischer Eingriffe und Kriege wiederholt Störungen unterworfen war. Ende der 1920er Jahre hatten sich die wirtschaftlicher Beziehungen wieder weitgehend normalisiert, d.h. Deutschland importierte aus dem Nachbarland vor allem landwirtschaftliche Güter, Wolle und Textilien, Erø und Eisenerzeugnisse; umgekehrt führte Frankreich hauptsächlich Kohle, Eisenund Metallerzeugnisse, Textil- und Werkzeugmaschinen, elektrotechnische Produkte und Holz ein. ${ }^{18}$ Allerdings war die Abhängigkeit Frankreichs wegen der nur schwer und recht kostspielig zu ersetzenden Ruhrkohle deutlich größer. Nach dem Zweiten Weltkrieg konzentrierte sich die französische Politik deshalb zunächst darauf, diese Abhängigkeit zu beseitigen oder wenigstens zu verringern, indem sie forderte, das Ruhrgebiet internationaler Kontrolle $\mathrm{zu}$ unterstellen. Nachdem dieses und andere einseitig-machtpolitischen Konzepte vor dem Hintergrund des Kalten Krieges und unter amerikanischem Druck zugunsten kooperativer Regelungen nach und nach aufgegeben werden mußten, verfolgte Frankreich eine Strategie der Kontrolle des westdeutschen Industriepotentials durch Integration und die Herstellung gleicher Ausgangsbedingungen insbesondere bei der Stahlproduktion, um auf dieser Grundlage zu den früheren Strukturen fruchtbarer wirtschaftlicher Zusammenarbeit zurückkehren zu können. ${ }^{19}$ Auch die deutsche Seite war verständlicherweise an einer raschen Wiederherstellung der wirtschaftlichen und finanziellen Beziehungen mit dem westlichen Nachbarn interessiert, allerdings unter der Bedingung der Gleichberechtigung. Der Chefunterhändler bei den Verhandlungen über das erste Handelsabkommen zwischen Bonn und Paris, Vollrath von Maltzan, vermochte dementsprechend schon im Januar 1950 kein anderes westeuropäisches Land zu erkennen, mit dem zusammenzuar-

18 Hermann BOHRER, Die deutsch-französischen Wirtschaftsbeziehungen und die Möglichkeiten ihrer zukünftigen Gestaltung, in: Europa-Archiv, 4 (1949), S. 2549-2556; Raymond POIDEVIN, Les relations économiques et financières entre la France et l'Allemagne de 1898 à 1914, Paris 1998.

19 Vgl. Rolf STEININGeR, Ein neues Land an Rhein und Ruhr. Die Ruhrfrage 1945/46 und die Entstehung Nordrhein-Westfalens, Köln 1990; Werner BÜHRER, Frankreich und das Ruhrgebiet - Mythos und Realität, in: WILKENS (Anm. 16), S. 225-236. 
beiten den Deutschen sannähernd gleiche wirtschaftliche Vorteile bringen könnte ${ }^{20}{ }^{20}$

$\mathrm{Zu}$ den besonderen Zielen der französischen Seite zählten zunächst die Sicherung des Kohlenbedarfs zu nicht-diskriminierenden Konditionen und eine Ausweitung der landwirtschaftlichen Exporte - vor allem Wein und Weizen - in die Bundesrepublik. Nach der Gründung der Europäischen Gemeinschaft für Kohle und Stahl (EGKS) gewann die Kanalisierung der Mosel, von der sich die französischen Stahlerzeuger eine Verbilligung der Kohle- und Koksimporte und gleichzeitig der Stahlexporte nach Süddeutschland erhofften, rasch an Dringlichkeit; die Erneuerung von Kartellabsprachen, die bis zu diesem Zeitpunkt ebenfalls weit oben rangierte, büßte dagegen zumindest im Montansektor an Bedeutung ein. Ferner sollte die Wiederkehr einer selbständigen deutschen Rüstungsindustrie verhindert werden, ebenso eine Orientierung der Wirtschaft der Bundesrepublik auf die früheren Absatzmärkte in Osteuropa und in der Sowjetunion. Als Gegenleistung bot man den Deutschen eine Zusammenarbeit bei der Durchfuihrung rüstungswirtschaftlicher Projekte - und zwar sowohl zur Produktion "klassischer" als auch moderner Rüstungsgüter - sowie eine schrittweise Öffnung der eigenen Märkte an. Schließlich drängte die französische Seite angesichts der durch die EGKS und später die EWG veränderten Wettbewerbsverhältnisse auf eine allmähliche Harmonisienung der Soziallasten - eine Forderung, die letztlich auf eine Erhöhung des deutschen Niveaus hinauslief. ${ }^{21}$

Für die Bundesregierung und die westdeutsche Wirtschaft stand, wie erwähnt, die Erlangung der wirtschaftlichen Gleichberechtigung ganz oben auf der Agen$\mathrm{da}$, und bei der Beseitigung der in den Jahren der Besatzung auferlegten alliierten Restriktionen hoffte man sogar auf Hilfe aus dem Nachbarland. Um diesen Beistand zu bekommen, war man zu begrenzten Zugeständnissen etwa bei französischen Anliegen wie der Moselkanalisierung oder beim Tempo der Handelsliberalisierung bereit. Der Abbau von Kontingenten und Zöllen diente allerdings nicht nur als "Einsatz« bei bilateralen Verhandlungen, sondern lag im ureigenen Interesse der Bundesrepublik mit ihrer hochgradig exportabhängigen Wirtschaft. Auf eine Erleichterung wirtschaftlicher Aktivitäten in Frankreich zielten auch die Forderungen nach Rückgabe der deutschen Warenzeichen und nach Niederlassungsfreiheit für deutsche Unternehmen - ein Anliegen, das selbst Mitte der S. 146-149, hier S. 148.

21 Vgl. mit zahlreichen Belegen BDFD 2 (Anm. 4); LEFĖVRE (Anm. 5); Raymond POIDEVIN, Jacques BARIÉTY, Frankreich und Deutschland. Die Geschichte ihrer Beziehungen 18151975, München 1982, S. 448-455. 
1960er Jahre noch nicht zu deren Zufriedenheit geregelt war. Und im Stahlsektor zeigten sich die Unternehmen an Rhein und Ruhr entschlossen, ihre "natürlichen« Standortvorteile, also die räumliche Nähe zur Kohle und zur Weiterverarbeitung, und die "Verbundwirtschaft", d.h. die durch entsprechende Eigentumsverhältnisse abgesicherte Verbindung von Kohlezechen und Stahlwerken hartnäckig zu verteidigen.

Beide Seiten waren indes bemüht, die gemeinsamen Interessen zu betonen: Dazu gehörten insbesondere die Intensivierung des Handels, der sich zwischen 1960 und 1970 in der Tat in etwa vervierfachte und auch eine Steigerung der französischen Fertigwarenexporte implizierte, sowie in zunehmendem Maße die Zusammenarbeit bei Projekten, etwa auf dem Gebiet der Rüstungsproduktion, des Flugzeugbaus, der Atomenergie und der Glasherstellung. Diese Vorhaben schlossen unter anderem die Entwicklung gemeinsamer Produktionsprogramme ein, ebenso die Suche nach Möglichkeiten zur Steigerung der Produktivität. Einen prominenten Platz nahm auch die Entwicklung gemeinsamer Vorhaben zur wirtschaftlichen Entwicklung Nordafrikas ein. ${ }^{22}$ Die geplante Kooperation erstreckte sich ferner auf die Aufteilung einzelner Märkte und die Vermeidung nüberflüssiger« Konkurrenz durch Übereinkünfte zwischen bestimmten Industriebranchen. Diese hauptsächlich bilateral ausgerichteten Pläne wurden mit der Intensivierung der Integration mehr und mehr überlagert von "europäischen " Zielen und der Absicht, gemeinsam eine Führungsrolle im Einigungsprozeß übernehmen zu wollen.

Einen guten Überblick über die jeweiligen Zielsetzungen ermöglichen die Treffen des deutsch-französischen Industrie-Komitees, zu denen BDI- und CNPFRepräsentanten in regelmäßigen Abständen zusammenkamen. Stand 1952 die Erörterung von Wegen zur Steigerung des Warenaustausches durch eine Multilateralisierung des Zahlungsverkehrs und einen raschen Übergang zur Konvertibilität der Währungen im Mittelpunkt, ${ }^{23}$ ging es zwei Jahre später um die Möglichkeiten für eine Intensivierung der Kooperation auf Branchenebene und um eine bessere Beteiligung der Wirtschaft an den Wirtschaftsverhandlungen zwischen den beiden Regierungen. ${ }^{24}$ Bei dem Treffen im Jahr 1956 wurde insbesondere über das Verhältnis des Komitees zur deutsch-französischen Handelskammer und zum gemischten, mit Regierungsvertretern besetzten Wirtschaftsausschuß sowie

Vgl. Sylvie LEFĖVRE, Projets franco-allemands de développement économique en Afrique du Nord (1950-1955), in: Revue d'Allemagne et des Pays de langue allemande, 25 (1993) 4, S. 581-588.

${ }^{23}$ Vgl. BDI-Jahresbericht 1951/52, S. 14.

24 Vgl. BDI-Jahresbericht 1954/55, S. 24. 
über ein neues Handelsabkommen debattiert. ${ }^{25}$ Mit der Unterzeichnung der Römischen Verträge und der Gründung der EWG rückte die Abstimmung in integrationspolitischen Fragen stärker in den Vordergrund. In dieser Phase waren die Beziehungen zwischen BDI und CNPF nicht frei von Spannungen, da die beiden Verbände unterschiedliche Konzepte verfolgten: Verstand der BDI den Gemeinsamen Markt lediglich als Zwischenschritt auf dem Weg zur Schaffung einer OEEC-weiten oder gar watlantischen « Freihandelszone, waren für den CNPF mit der EWG bereits die "Grenzen der Belastbarkeit« der heimischen Wirtschaft erreicht. ${ }^{26}$ Der BDI erblickte deshalb seine »große Aufgabe « darin, zwischen den europapolitisch wauseinanderstrebenden Auffassungen insbesondere der Franzosen und der Engländer einen Ausgleich « zu finden. ${ }^{27}$ Dieses Thema blieb zunächst auf der bilateralen Agenda: 1962 wurde neben den Perspektiven des Gemeinsamen Marktes und den Beziehungen zu dritten Ländern auch der Beitritt Großbritanniens zur EWG erörtert. ${ }^{28}$ Erst 1963, nach dem Scheitern des britischen Beitritts, wandten sich die beiden Delegationen neuen Problemen zu, beispielsweise der Notwendigkeit einer "verstärkten Abstimmung in wettbewerbs-, steuer- und agrarpolitischen Fragen«, der Möglichkeit von deutschen Investitionen in ehemals französischen Entwicklungsländern und den bevorstehenden Zollverhandlungen im Rahmen des GATT. ${ }^{29}$ Obwohl bei diesen Treffen nicht immer ein Konsens erzielt werden konnte, vermittelten sie doch hilfreiche Einblicke in die Vorstellungen und Absichten der Gegenseite und halfen mit, das gegenseitige Verständnis zu vertiefen.

\section{Zum Einfluß wirtschaftlicher Akteure auf die deutsch-französische Zusammenarbeit}

Den Einfluß der beiden Spitzenverbände und der anderen wirtschaftlichen Akteure auf die Entwicklung der Zusammenarbeit zwischen der Bundesrepublik Deutschland und Frankreich bis zum Elysée-Vertrag zu bewerten, erscheint allenfalls im Einzelfall möglich. Generell läßt sich festhalten, daß die deutsche Wirt-

\footnotetext{
Vgl. BDI-Jahresbericht 1956/57, S. 30.

Zahlreiche Belege in BDFD 2, das Zitat in WILKENS (Anm. 4), S. 45.

Protokoll Präsidialsitzung vom 20.3.1958, BDI-Archiv, HGF Pro, 5.

Vgl. BDI-Jahresbericht 1961/62, S. 34.

Vgl. BDI-Jahresbericht 1963/64, S. 34.
} 
schaft einen größeren Einfluß ausgeübt zu haben scheint als die französische. Diesen Schluß erlauben jedenfalls die wiederholten Klagen der französischen Industrie über eine unzureichende Beteiligung an den Verhandlungen sowohl über den Schuman-Plan als auch über den Gemeinsamen Markt; zwar beschwerten sich ihre deutschen Kollegen ebenfalls, doch hatten sie zumindest im ersteren Fall kaum Grund dazu. Sofern sich die jeweilige Regierung jedoch dezidiert für eine bestimmte Politik entschieden hatte, vermochten weder die deutschen noch französischen Unternehmer daran etwas zu ändern: Weder konnten etwa die französischen Stahlindustriellen die Montanunion verhindern, noch die deutsche Industrie insgesamt die EWG zugunsten einer Freihandelszone. ${ }^{30}$

Wenn hingegen eher technische Probleme zu klären waren - beispielsweise die Rückgabe der Warenzeichen, der Abbau von Kontingenten oder die Implikationen der sozialen Harmonisierung - fanden Forderungen einzelner Verbände teilweise wörtlich Eingang in Regierungsdokumente. Auf diesen Feldern konnten sie ihr Fachwissen ausspielen, auf das die Bürokratien gerade in Bonn, aber auch in Paris mitunter dringend angewiesen waren. Auch im Fall der europäischen Integration gilt es zu unterscheiden: Sofern die Vorstellungen und Wünsche von Unternehmern und Unternehmerverbänden die Regierungspolitik unterstützten, war die Chance, da 3 sie in offiziellen Verhandlungspapieren ihren Niederschlag fanden, recht groß. Es waren also keine eminent politischen Angelegenheiten, bei denen sich eine Einflußnahme nachweisen läßt. Dennoch sollte die natmosphärische" Bedeutung intensiver und vertrauensvoller Kontakte gerade zwischen hochrangigen Unternehmern dies- und jenseits des Rheins nicht unterschätzt werden. Nicht nur in den Anfangsjahren der deutsch-französischen Verständigung, als die Möglichkeiten direkter "politischer« Kontakte noch gering waren, spielten sie eine wichtige Rolle. Auch in späteren "kritischen" Momenten der deutschfranzösischen Beziehungen dienten sie dazu, Standpunkte zu klären und Probleme zu entschärfen. Und manchmal erteilten insbesondere die beiden Spitzenverbände sogar wohlmeinende Ratschläge an die Politik - eine Praxis, an die der BDI und sein französischer Partnerverband, der inzwischen unter dem Namen MEDEF firmiert, anläßlich des 40. Jahrestages des deutsch-französischen Vertra-

Vgl. Werner BÜHRER, Ruhrstahl und Europa. Die Wirtschaftsvereinigung Eisen- und Stahlindustrie und die Anfänge der europäischen Integration 1945-1952, München 1986, S. 185215; Matthias KIPPING, Zwischen Kartellen und Konkurrenz. Der Schuman-Plan und die Ursprünge der europäischen Einigung 1944-1952, Berlin 1996; Thomas RHENISCH, Europäische Integration und industrielles Interesse. Die deutsche Industrie und die Gründung der Europäischen Wirtschaftsgemeinschaft, Stuttgart 1999; MOGUEN-TOURSEL (Anm. 12). 
ges anknüpften. ${ }^{31}$ Das Beziehungsgeflecht aus Verbänden, Unternehmen und Unternehmern bildete jedenfalls ein enorm strapazierfähiges Fundament für die dauerhafte Aussöhnung und Zusammenarbeit zwischen den einstigen »Erbfeinden«. Das Fehlen substantieller wirtschaftlichen Bestimmungen im ElyséeVertrag läßt sich deshalb zwar auch damit erklären, daß die entscheidenden Protagonisten - Adenauer und de Gaulle - wirtschaftlichen Fragen seit jeher keine große Beachtung schenkten; wichtiger war jedoch, daß die wirtschaftliche $\mathrm{Zu}$ sammenarbeit bereits so gut funktionierte, daß zusätzliche vertragliche Vereinbarungen überflüssig waren.

${ }^{31}$ Pressemitteilung des BDI und der Bundesvereinigung der Deutschen Arbeitgeberverbände, 19.1.2003. 
